\title{
Power Control for Stable Achievement of Local Hardening of Steel Using Rapid Resistance Heating
}

\author{
Seijiro Maki ${ }^{1{ }^{* *},}$ Yuuki Makida ${ }^{2}$, Eitoku Nakanishi $^{1}$ \\ ${ }^{1}$ Division of Mechanical Engineering, Graduate School of Engineering, Mie University, Japan \\ ${ }^{2}$ Mie University, Japan
}

Copyright (C) 2015 by authors, all rights reserved. Authors agree that this article remains permanently open access under the terms of the Creative Commons Attribution License 4.0 International License

\begin{abstract}
The method of local hardening of steel using rapid resistance heating was devised by focusing attention on one of the unique characteristics of the resistance heating that the heat generated by Joule heating per unit volume at the area in contact with electrodes is directly proportional to the square of current density and inversely proportional to the contact area ratio. This characteristic enables heating of steel for austenitizing essential to hardening even at the area in contact with the electrode of high thermal conductivity. In this study, effective measures for power control were considered to ensure stable achievement of local hardening in the case that there is an impedance change due to change of the treating objects or the treating positions in just the same object. A measure conclusively suggested as a strongly possible one was input heat control based on the integrated value of square of flowing current with respect to time, relating to the resistance heating characteristic in the present case. Effectiveness of the control was confirmed by experiments with an alternating current power supply to which the function described above was added.
\end{abstract}

Keywords Heat Treatment, Hardening, Local Hardening, Resistance Heating, Power Control

\section{Introduction}

Though there have been various kinds of material processing which make use of resistance heating, that is, Joule heating by direct energizing, its application to metal-based powder sintering by F.V. Lenel [1] would be a pioneer work if applications in welding field were excepted. Since then, its application has been expanding outside the field of sintering, and now it is extended to hot stamping and die-quenching of steel sheet for automobile parts production which needs heating sheet materials [2, 3]. In these circumstances, one of the authors also has researched into its application to fabrication of metal matrix composites and their simultaneous forming and joining to metal parts in the fabricating process [4, 5]. And further, relating to mushy-state forming or semi-liquid forming of metallic materials, the resistance heating technique has been applied to the forming process for aluminum alloy A357 [6] and magnesium alloy [7]. The method for local hardening of steel parts was thought up by focusing attention on a unique characteristic of resistance heating at the electrode contact area, and successful performance of local hardening was demonstrated by application of proper resistance heating condition [8]. In this study, with a view to stable achievement of local hardening in use of an inexpensive $\mathrm{AC}$ power supply, the heating characteristic at the electrode contact area was considered and an effective measure was devised as to power control.

\section{Resistance Heating Characteristic at Electrode Contact Area}

In the method for local hardening using rapid resistance heating, a cylindrical electrode is imposed upon a treating surface and electricity is conducted through it to resistance-heat the area where the electrode is in contact. To think of heating of the area in contact with the electrode at any moment, a nominal current density $i$ passing through the area is given as $i=I / S$, where $I$ is an electric current and $S$ is a nominal contact area of the electrode. A contact of the electrode with the treating material is not complete but partial in area. To express the contact area ratio by $\varepsilon$ and the electrical resistivity of the treating material by $\rho$, the averaged amount of heat generated at the contact area in unit volume and unit time, $q$, can be given as follows.

$$
q=\frac{\rho}{\varepsilon} i^{2}=\frac{\rho}{\varepsilon S^{2}} I^{2}
$$

The contact area ratio $\varepsilon$ depends not only on the contact pressure $p_{c}$ applied to the electrode but also on the yield stress of the softer material in contact. The yield stress and the electrical resistivity $\rho$ both vary with the temperature. Therefore, when the contact pressure $p_{c}$ is fixed, the contact area ratio $\varepsilon$ and the electrical resistivity $\rho$ become physical quantities which only depend on the temperature not on the heating rate. Consequently, the heat $q$ generated at the 
electrode contact area in the treating material per unit volume and unit time is in proportion to the square of electric current density, $i^{2}$, as shown by Eq. (1) and the total amount of generated heat $Q$ at the contact area is expected to be a close relation to the integrated value of the square of flowing electric current with respect to time. And this implies that stable achievement of local hardening will be realized by control of input heat $Q$ through proper setting of the integrated value $\int I^{2} d t$.

\section{Experimental Procedure}

Materials. Cold die steel (SKD11) rod with a diameter of $32 \mathrm{~mm}$ which can be hardened by air-cooling from austenitizing temperature was used as an experimental material. The rod was cut into pieces with a length of $20 \mathrm{~mm}$ and cut surfaces of the pieces were buff-finished. And the local hardening treatment was given on the surfaces. As for the electrode, a composite alloy of copper and tungsten, EM-C30A2, containing 30 mass \% of copper, produced by Nippon Tungsten Co., Ltd. was used taking its durability into consideration [9].

Apparatus. Figure 1(a) shows a schematic of the local hardening method using resistance heating. In the experiments an electrode with a tip diameter of $5 \mathrm{~mm}$ shown in Figure 1(b) was pressed on the test piece at a predetermined pressure with an air cylinder and the test piece was electrified for a predetermined time to heat. The electrode was let stand for $5 \mathrm{~s}$ after resistance heating so as to cool the heated area of the test piece. Output impedance of the power supply used for the experiments was about 2 $\mathrm{m} \Omega$ on the load between the copper plates shown in Fig. 1(a). Amperage during the resistance heating was measured with an interval of $0.5 \mathrm{~ms}$ and recorded in order to obtain the integrated value of the square of the electric current with respect to time.

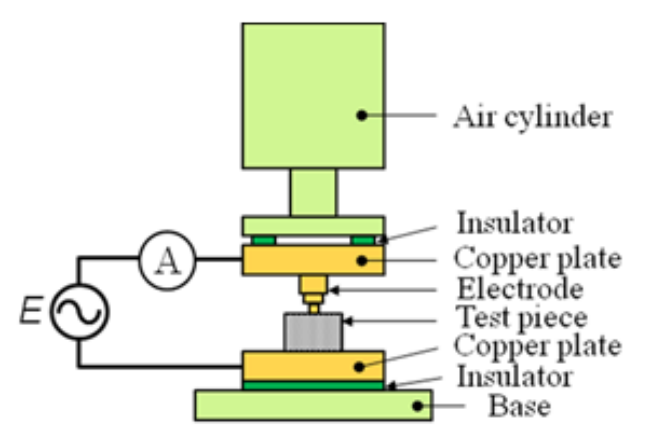

(a)

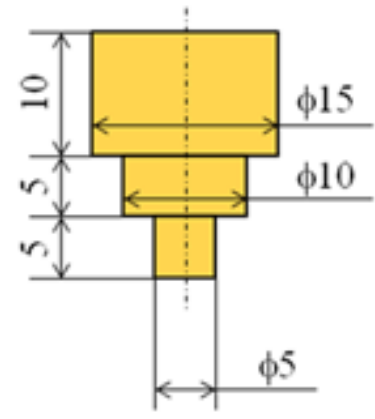

(b)

Figure 1. Illustrations of (a) method of local hardening using resistance heating and (b) electrode used for experiment.

\section{Results and Discussion}

Influence of resistance heating condition on hardening performance. Figure 2 shows photographs of test piece surfaces after the treatment which was given to the test pieces on the conditions of electrode contact pressure $p_{c}=$ $21.8 \mathrm{MPa}$, current supply time $t=1 \mathrm{~s}$ and applied voltage $E$ $=6,7$ and $8 \mathrm{~V}$. It is found that a circularly heated area expands by an increase of the applied voltage or an increase of input heat. Figure 3 shows Vickers hardness distribution of the treated area. It is seen that a hardened area initially develops circularly a little apart from the heating center, and that an increase of the applied voltage, that is, an increase of input heat makes the hardness at the center increase. At the applied voltage $E=8 \mathrm{~V}$, the hardness at the center becomes the same level as that at the periphery, indicating that hardening was uniformly achieved. From the fact above, in the experiments described below, the condition of hardening was ranked according to the hardness at the heating center.
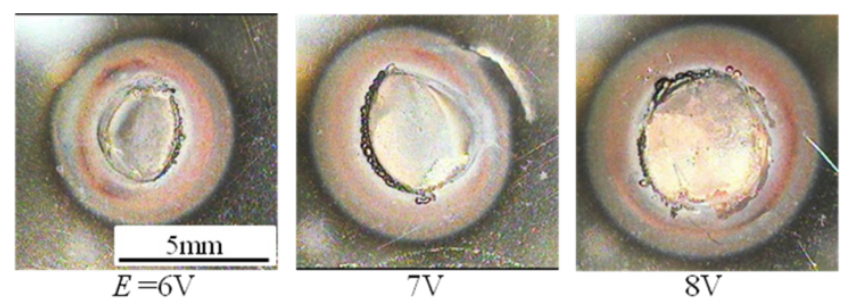

Figure 2. Changes in condition of surface with applied voltage $E$. (Electrode contact pressure $p_{c}=21.8 \mathrm{MPa}$ and current supply time $t=1.0 \mathrm{~s}$ ) 


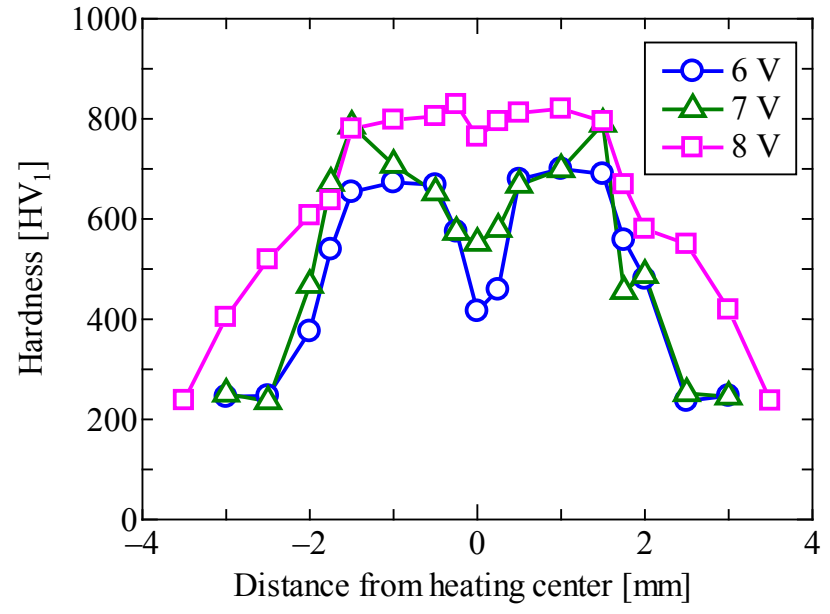

Figure 3. Variation of hardness distribution with applied voltage $E$. (Electrode contact pressure $p_{c}=21.8 \mathrm{MPa}$ and current supply time $t=1.0 \mathrm{~s}$ )
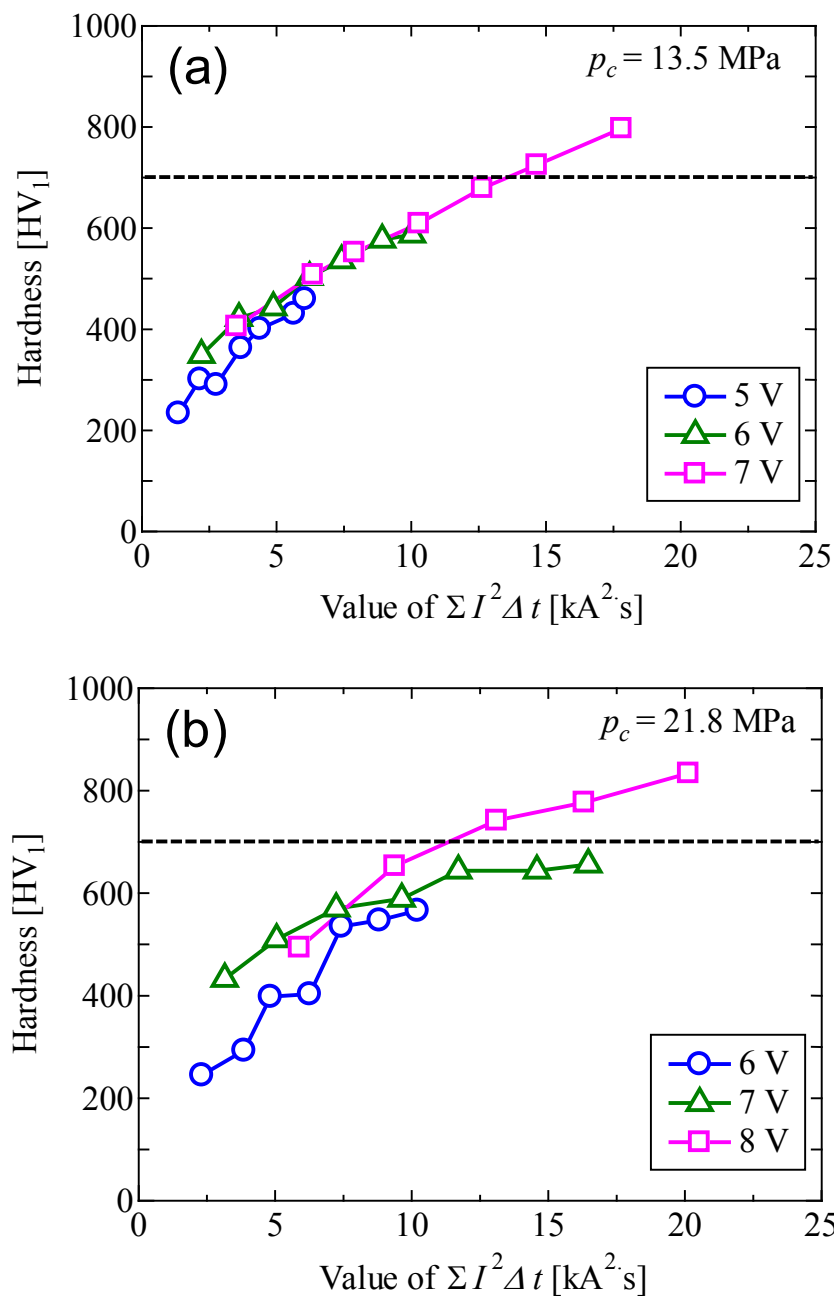

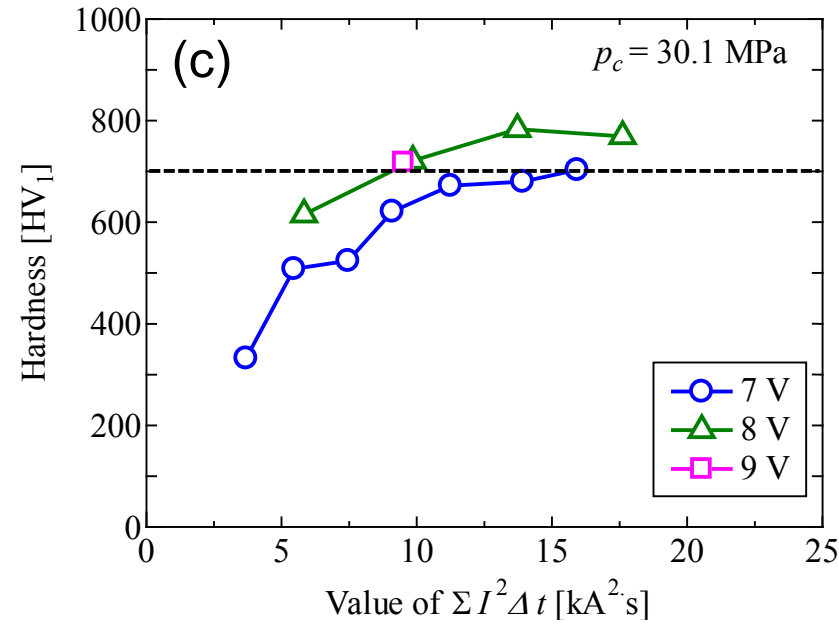

Figure 4. Variation of hardness at heating center with value of $\sum I^{2} \Delta t$ : (a) Electrode contact pressure $p_{c}=13.5 \mathrm{MPa},(\mathrm{b}) p_{c}=21.8 \mathrm{MPa}$ and (c) $p_{c}=30$. $1 \mathrm{MPa}$.

Relationship between hardness at heating center and integrated value of square of current with respect to time. Figure 4 shows the relation between the hardness at the heating center and the accumulated value $\sum I^{2} \Delta t\left[\mathrm{kA}^{2} \cdot \mathrm{s}\right]$ of the product of the square of electric current $I$ and time interval $\Delta t$. These results were obtained from the experiments as follows. At three levels of the electrode contact pressure $p_{c}, 13.5 \mathrm{MPa}, 21.8 \mathrm{MPa}$ and 30.1 $\mathrm{MPa}$, the applied voltage $E$ was set at three levels in the range from 5 $\mathrm{V}$ to $9 \mathrm{~V}$ by the step of $1 \mathrm{~V}$, and the test piece was electrified in time-control. The current supply time was set in the range from $0.5 \mathrm{~s}$ to $2.0 \mathrm{~s}$ by step of $0.25 \mathrm{~s}$. The leftmost data point in each curve corresponds to the result for the current supply time $0.5 \mathrm{~s}$. To think of the practical application, the treatment which accompanies surface damage should be avoided. In this connection, the results in which melt adhesion of the electrode occurred were deleted even if the preset current supply time was less than $2.0 \mathrm{~s}$. In addition, in the experiment, the hardness level $700 \mathrm{HV}_{1}$ was set as a judgmental standard for ranking the hardening performance.

Hardening performance in power control with integrated value of square of current with respect to time, $\int I^{2} d t$. In order to realize the power control by the integrated value of square of electric current with respect to time, an AC power supply having time control and input energy control functions was improved by adding a switch so as to select a current signal in place of a voltage signal for making a square of the current as shown in Figure 5. Hereafter, the power control by the integrated value will be described as $\int I^{2} d t$-control in short. 


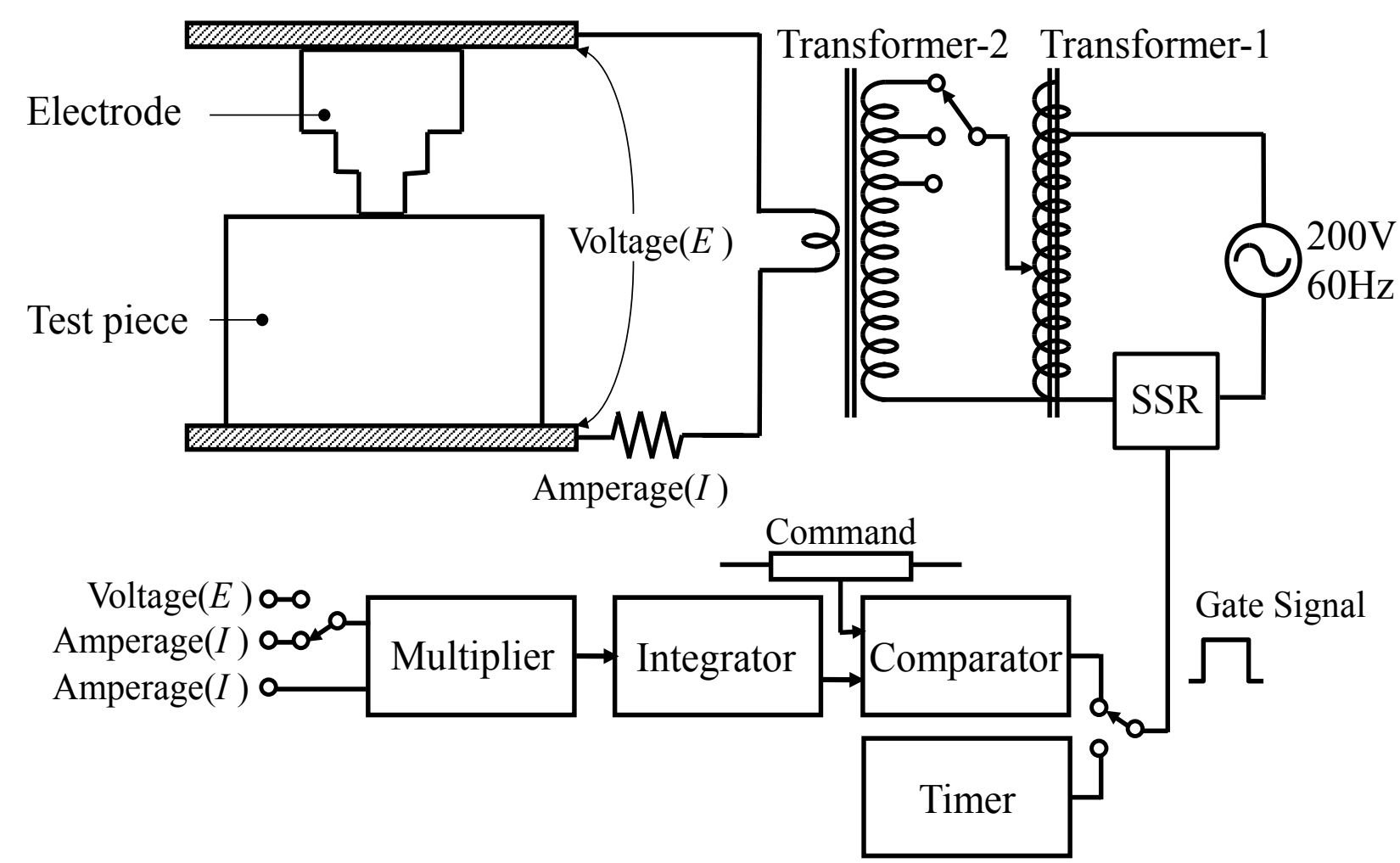

Figure 5. Experimental setup for local hardening using AC power supply in which power control by integrated value of square of current with respect to time, $\int I^{2} d t$-control, is built.

Table 1. Comparison of value of $\sum I^{2} \Delta t\left[\mathrm{kA}^{2} \cdot \mathrm{s}\right]$ actually observed in $\int I$ ${ }^{2} d t$-controlled resistance heating with set value at some electrode contact pressures $p_{c}$. (Applied voltage $E=8 \mathrm{~V}$ )

\begin{tabular}{|c|c|c|c|}
\hline & $p_{c}=13.5 \mathrm{MPa}$ & $p_{c}=21.8 \mathrm{MPa}$ & $p_{c}=30.1 \mathrm{MPa}$ \\
\hline $\begin{array}{c}\text { Set value of } \sum I^{2} \Delta t \\
{\left[\mathrm{kA}^{2} \cdot \mathrm{s}\right]}\end{array}$ & 12.3 & 13.6 & 13.4 \\
\hline $\begin{array}{c}\text { Observed in } \int I \\
{ }^{2} d t \text {-control }-1\end{array}$ & 12.5 & 13.7 & 13.3 \\
\hline $\begin{array}{c}\text { Observed in } \int I \\
{ }^{2} d t \text {-control - } 2\end{array}$ & 12.5 & 13.6 & 13.4 \\
\hline
\end{tabular}

As shown in Fig. 5, the $\int I^{2} d t$-controller added to the AC power supply is composed of multiplier, integrator and comparator so as to have a function to complete the current supply as soon as the integrated value of the square of current with respect to time exceeds the value preset as a command. Using the setup in Fig. 5, preliminary experiments were conducted to certify the performance of the controller and reproducibility. In the experiments, the hardening treatment by time-controlled resistance heating was firstly done under the conditions of the applied voltage $E=8 \mathrm{~V}$ and electrode contact pressure $p_{c}=13.5 \mathrm{MPa}, 21.8 \mathrm{MPa}$ and $30.1 \mathrm{MPa}$, which assured satisfactory hardening by current supply for 1 $\mathrm{s}$ as shown in Fig. 4, and hardness distribution in the treated area was examined in relation to the value of $\sum I^{2} \Delta t$. Then, by using the value of $\sum I^{2} \Delta t$ obtained from the preliminary experiment as a set value, experiment of the $\int I^{2} d t$-control was carried out. Table 1 shows comparison of the value of $\sum I^{2} \Delta t$ between actually observed and set ones. In each case, an observed value was found to be nearly the same as a set value.

Figure 6 shows the hardness distributions in the case of the $\int I^{2} d t$-controlled resistance heating compared with that in the case of the time-controlled resistance heating. Somewhat difference was found, but the value of Vickers hardness and the extent of hardened area are almost the same between the two cases. From these facts, it can be concluded that the power control based on the value of $\int I^{2} d t$ is effective for stable achievement of the local hardening using rapid resistance heating.

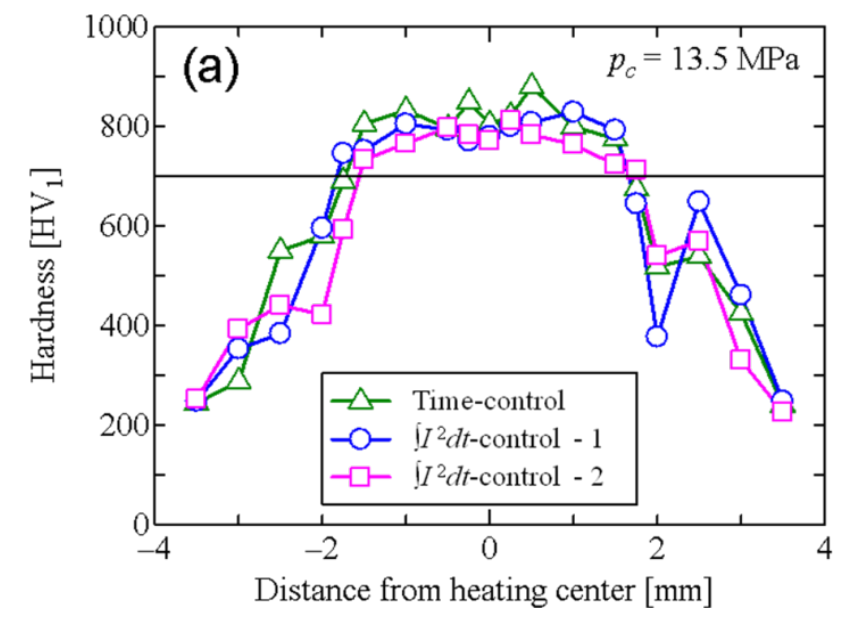



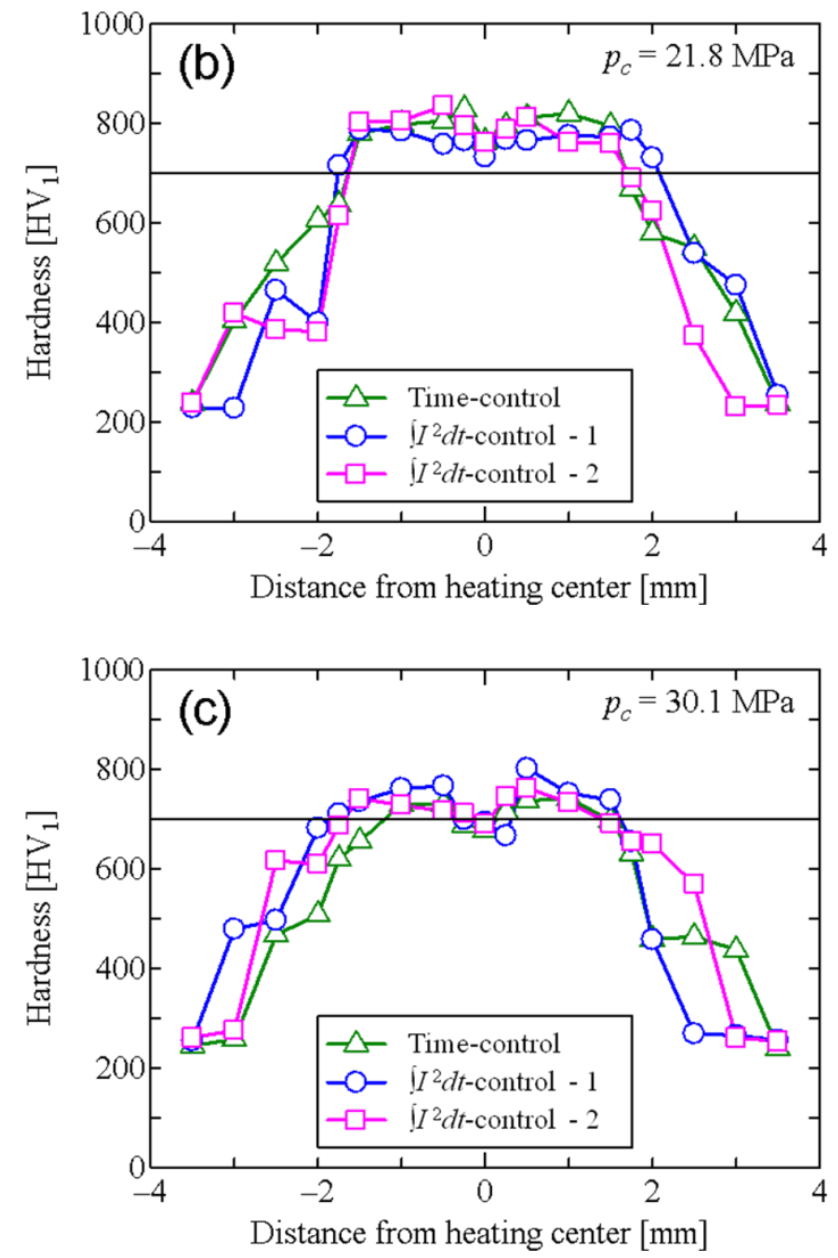

Figure 6. Comparison of hardness distribution between two control modes, $\int I^{2} d t$-control and time-control. Optimum set value $\sum I^{2} \Delta t$ in each case was extracted from just the time-controlled resistance heating shown for comparison. (Applied voltage $E=8 \mathrm{~V}$ )

As for the setup shown in Fig. 5, an impedance change will occur by shift of treating position. When impedance is increased, flowing current decreases, and vice versa. Then, on the assumption that there is an increase of impedance or a decrease of flowing current in operation of local hardening, the influence of a decrease in flowing current on hardening performance was examined by experiment. The decrease of the floating current was realized by decreasing the applied voltage from $8 \mathrm{~V}$ to $7 \mathrm{~V}$, and resistance heating in the mode of $\int I^{2} d t$-control was done under the condition that the value $\sum I^{2} \Delta t$ and the electrode contact pressure $p_{c}$ were both fixed. According to Ohm's and Joule's laws, the current supply time of $1 \mathrm{~s}$ at the applied voltage of $8 \mathrm{~V}$ is expected to be changed into $1.31 \mathrm{~s}$ at $7 \mathrm{~V}$. Actually observed current supply time was in the range 1.3 to $1.5 \mathrm{~s}$ as was expected. Figure 7 shows the hardness distribution obtained from the $\int I$ ${ }^{2} d t$-controlled resistance heating $(E=7 \mathrm{~V})$, compared with that obtained from the time-controlled resistance heating ( $E$ $=8 \mathrm{~V}$ ), under the condition of the electrode contact pressure $p_{c}=13.5 \mathrm{MPa}$ and $21.8 \mathrm{MPa}$. To compare the results obtained from the $\int I^{2} d t$ - controlled resistance heating in Fig. 7(a) and Fig. 6(a), the hardness in Fig. 7(a) is lower than that in Fig. 6(a) whose current supply time was shorter due to the higher applied voltage. The situation between Fig. 7(b) and Fig. 6(b) is almost the same. As seen from these comparisons, even in the case that the set values of $\sum I^{2} \Delta t$ are same, there is fear of not obtaining satisfactory hardening when the current supply time actually required is too long. The unsatisfactory hardening is attributed to insufficient austenitizing which is caused by a relative increase of heat loss by conduction for the long heating time. Therefore, on the occasion when $\int I^{2} d t$ control is put to use, attention should be paid to the actually required current supply time in relation to the current supply time which is specified in the examination of the value of $\sum I^{2} \Delta t$

In connection with the $\int I^{2} d t$-control, if a DC power supply having a constant current regulator is available, there is no necessity of incorporating such a controller because proper heating is easily realized by adjusting the current intensity to meet the command, the set value of $\sum I^{2} \Delta t$ including predetermined current supply time.
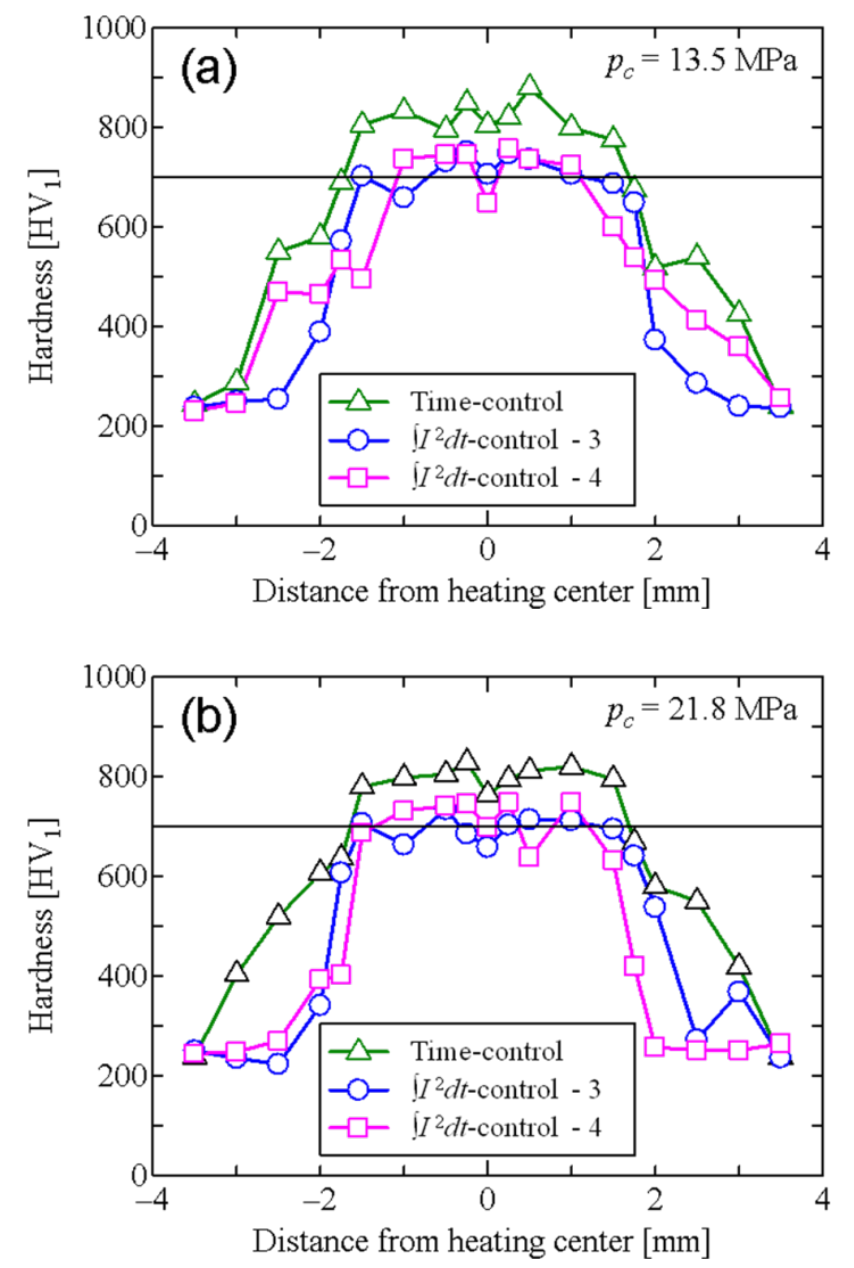

Figure 7. Comparison of hardness distribution obtained from $\int I$ ${ }^{2} d t$-controlled resistance heating at optimum set value of $\sum I^{2} \Delta t$ with that from time-controlled resistance heating optimally conditioned, to show influence of increased impedance or decreased current on hardening performance. ( $E=8 \mathrm{~V}$ (time-control), $7 \mathrm{~V}\left(\int I^{2} d t\right.$-control)) 


\section{Conclusions}

As for the local hardening treatment using resistance heating the effectiveness of power control by the integrated value of square of electric current with respect to time was examined using cold die steel, SKD11, as an experimental treating material, and the followings were found.

(1) Heat input to the treating material is strongly related to the integrated value of the square of electric current with respect to time and an increase of the value increases the hardness of the treated area in a proper range of the value.

(2) Power control by the integrated value is effective for stable achievement of the hardening treatment when the current supply time actually required is in the allowable range.

(3) In connection with the control, in the case that the current supply time actually required for heating is much more than the predetermined time, satisfactory hardening is not obtained because of insufficient heating for austenitizing due to a relative increase of heat loss by conduction for the long heating period, and adversely in the case that the current supply time actually required is much less than the predetermined time, surface damage would be caused due to an excessive current density.

(4) As for the countermeasure to the matter (3), in the practical application, an alarm system should be added to the controller to put out an alert when the current supply time actually required is not in the allowable range.

\section{Acknowledgements}

This work was supported by JSPS KAKENHI Grant Number 24560877 and the Amada Foundation Grant Number AF-2010005.

\section{REFERENCES}

[1] F.V. Lenel: Trans. AIME Vol. 230 (1955), 158-167.

[2] S. Maki, A. Hamamoto, S. Saito and K. Mori: Key Engineering Materials Vol. 344 (2007), 309-316.

[3] S. Maki, Y. Kusunoki and E. Nakanishi: Advanced Materials Research Vol. 939 (2014), 422-429.

[4] S. Maki, Y. Harada and K. Mori: Journal of Materials Processing Technology Vol. 143-144 (2003), 561-566.

[5] S. Maki, Y. Harada and K. Mori: International Journal of Materials and Product Technology, Special Issue, SPM1, 2 (2001), 512-517.

[6] S. Maki, Y. Harada, K. Mori and H. Makino: Journal of Materials Processing Technology Vol. 125-126 (2002), 477-482.

[7] S. Maki, Y. Harada and H. Makino: Mater. Sci. Forum Vol. 419-422 (2003), 387-392.

[8] S. Maki, S. Matsuda and E. Nakanishi: Proceedings of the 15th International Conference on Advances in Materials \& Processing Technologies, USB Flash, (2012), ORP-11418.

[9] Information on http://www.nittan.co.jp 